\title{
La percepción sobre la función de la comunicación corporativa en los sectores tradicionales
}

\author{
Rocío Blay Arráez ${ }^{1}$ \\ Maria Teresa Benlloch Osuna ${ }^{2}$ \\ Guillermo Sanahuja Peris ${ }^{3}$ \\ Universitat Jaume I
}

Recibido: 02/01/2014

Aceptado: 03/06/2014

\begin{abstract}
Resumen
Esta investigación tiene como objeto conocer la percepción sobre la función de la comunicación corporativa en los sectores tradicionales a partir de la estructura de los propios departamentos y de la consideración hacía el perfil del Dircom. Tras profundizar en los pilares teóricos del corporate y en la casuística de estos sectores tradicionales de la Comunidad Valenciana (clusters de la agroalimentación, calzado, cerámica, iluminación, juguete, mueble y textil) se desarrolla una metodología cualitativa en la que han participado a profesionales de estos sectores, representantes de las asociaciones sectoriales y profesionales de la comunicación en la comunidad valenciana. La principal conclusión extraída a lo largo de la investigación es que los sectores objeto de estudio no conocen y, por tanto, no confían en las posibilidades de una función estratégica de la comunicación en el seno de sus organizaciones. Rechazan la idea de que la imagen corporativa, correctamente gestionada, es un activo estratégico al servicio de cualquier organización, prevaleciendo la visión cortoplacista y la función comercial y de marketing.
\end{abstract}

Palabras clave: comunicación; departamento; sectores empresariales tradicionales.

\section{Perception of the corporate communication role in the traditional sectors}

\begin{abstract}
The purpose of this research is to gain knowledge on the perception about the corporate communication role in the traditional sectors, coming from its own departmental structure and considering its communication manager profile. After a deep analysis on the theoretical corporate pillars and the casuistry of these traditional sectors (clusters of agricultural engineering, footwear, ceramics, lighting, toys, furniture and textile), it is developed a qualitative methodology with the collaboration of professionals from these sectors, representatives of the sectorial associations and professionals of communication in the comunidad valenciana. The principal conclusion obtained during the research is that the studied sectors do not know and, therefore, do not trust the possibilities of a strategic role of communication inside their organizations. They reject the idea that the corporate image, correctly managed, is a strategic asset in service for any organization, prevailing over the short term vision and the commercial and marketing role.

Keywords: corporate communication; departmental structure; traditional business sectors.

${ }^{1}$ La doctora Rocío Blay Arráez es profesora colaboradora en la Universitat Jaume I. rblay@, com.uji.es

${ }^{2}$ La doctora Mayte Benlloch Osuna es profesora colaboradora en la Universitat Jaume I. ben1loch@,com.uji.es

${ }^{3}$ El doctor Guillermo Sanahuja Peris es profesor asociado en la Universitat Jaume I y director de Proyectos en Sanahuja \& Gimeno.gsanahuj@ com.uji.es
\end{abstract}




\section{Introducción: El interés de esta investigación}

Los sectores tradicionales mantienen una fuerte penetración en la geografía española. Esta investigación que centra su foco en la Comunidad Valenciana presenta unas conclusiones que son extrapolables a otros sectores tradicionales y a otros territorios por la homogeneidad en sus estructuras.

Los sectores tradicionales de la Comunidad Valenciana (agroalimentación, calzado, cerámica, iluminación, juguete, mueble y textil) son auténticos clúster ${ }^{4}$ generadores de empleo y riqueza de hecho, por su alto grado de concentración geográfica, hay poblaciones donde más del $50 \%$ del empleo viene generado por ellos. Sin embargo, atraviesan desde hace años por una situación crítica (PLA BARBER, J. et al, 2007), ya la Cámara de Comercio de Valencia publicó en 2004 un informe elaborado por la Universidad Politécnica, donde retrataban la difícil situación que estaban atravesando y reclamaban por parte de las administraciones públicas iniciativas concretas para frenar la inercia de cierre de empresas y pérdida de empleo.

Este artículo, como continuación de anteriores comunicaciones publicadas entre 2006 y 2010 (Blay Arráez, R. y Benlloch Osuna, Mª T, 2006, 2007, 2010; y Benlloch Osuna, $\mathrm{M}^{\mathrm{a}} \mathrm{T}$ y López Lita, R, 2005, 2006), considera que el conocimiento de la función de la comunicación en estos sectores y la importancia que se le atribuye es clave para revelar las carencias de estas organizaciones en el contexto actual de la sociedad de la información y, a continuación, proponer planes que permitan la gestión de la marca y la creación y el mantenimiento de una imagen de marca como factores clave para el futuro y supervivencia de los mismos.

\section{El marco teórico}

\subsection{La comunicación corporativa como activo estratégico empresarial}

En la actualidad muchas corporaciones ya han tomado este nuevo rumbo que era necesario y tiene que ver más, con una nueva sensibilidad empresarial, donde se pretende revertir parte del beneficio que tienen las empresas a la sociedad donde desarrolla su actividad y conjugar los intereses empresariales con los de los públicos con los que se relaciona (Costa, 2008). La aceptación general de la responsabilidad pública que tienen las organizaciones es un hecho vital y son conscientes a partir de este momento, que tienen que demostrar un comportamiento socialmente responsable, con el objeto de

\footnotetext{
${ }^{4}$ Los clusters, son grupos internamente homogéneos, pero distintos entre sí, bien por agrupación de unidades más pequeñas o por división de segmentos mayores, se ha aplicado en marketing especialmente en segmentación de mercados. Un cluster en el mundo industrial es una concentración de empresas relacionadas entre sí, en una zona geográfica relativamente definida, que conforman en sí mismas, un polo productivo especializado con ventajas competitivas. Este concepto fue popularizado por el economista Michael Porterel año 1990, en su libro The Competitive Advantage of Nations ("Ventaja Competitiva de las Naciones"). SANTESMASES MESTRE, M. (1996), pág. 67. Giuliani y Rabellotti, afirman que las empresas de un cluster tienen un comportamiento común en términos de orientación exportadora, subcontratación de algunas fases de la producción, estrategia inversora, nivel tecnológico o calidad de los productos, etc. y esto, es lo que sucede entre los sectores tradicionales de la Comunidad Valenciana. GIULIANI, E. y RABELLOTTI, R. (Edit.) (2005).
} 
obtener ahora sí la plena confianza de la opinión pública. El modo más directo de ganar el reconocimiento del público estriba en abrir canales de comunicación constantes y bidireccionales (Capriotti, 1992) con los públicos, y fomentar así, la participación de los ciudadanos en el destino de la organización.

Con la revolución de los servicios, de la que habla Joan Costa (2003), dentro de todos estos cambios en la filosofía empresarial también debe ser apuntado como las personas sustituyen a las máquinas y pasan al primer rango de la relación directa dentro de la organización y con los clientes, donde antes habían estado los productos de consumo. Los servicios son las personas, servir es lo contrario de dominar. En esta nueva perspectiva, el «cara a cara» ya no es el del consumidor con el producto inerte. Es el cliente con el empleado, es decir, con la empresa, en la medida que éste la representa explícitamente y es parte de ella. Con las personas en primer plano en la cultura de servicio, las empresas redescubren los recursos humanos, y con ello, se impone la exigencia de programas de calidad, la cultura organizacional con la gestión de carreras, y la comunicación interna como agente integrador. Además del ascenso del «corporate» ya que toda esta serie de transformaciones que ha arrastrado la revolución de los servicios puso el acento en la institución y en la estrategia corporativa, o sea global, del negocio. Así, los productos y las marcas se han quedado bien visiblemente circunscritos al campo del consumo. La imagen ocupa el primer plano. Primero fue la reafirmación de la identidad corporativa: la empresa como realidad única, total e irrepetible. Es el ascenso del espíritu institucional y la gestión integrada de los recursos. Es la irrupción revolucionaria de los intangibles y los valores (Pizzolante Negrón, 2006).

Dentro de todo este cambio de filosofía organizacional se asume que toda actuación de la empresa está comunicando, incluida la inactividad en este terreno, por tanto, la comunicación corporativa se convierte en un activo estratégico empresarial que debe ser gestionado. Además, a este se le confiere la posición de eje vertebrador del resto de activos estratégicos empresariales por su carácter transversal (Costa, 2001). Por tanto, dispone bajo su paraguas, todas las actuaciones de una organización, sean de la índole que sean, como determinantes en la configuración de la imagen corporativa, considerada esta como el fin último que persigue la comunicación corporativa, alcanzar una imagen positiva. Si además esta imagen positiva, es mantenida en el tiempo, con un comportamiento corporativo excelente y se satisfacen las expectativas de los "stakeholders», se convertirá en reputación corporativa, fin último que debe alcanzar la empresa actualmente para garantizar su permanencia (Villafañe, 2004). La reputación corporativa es considerada como el activo de mayor capacidad de diferenciación y el que más valor puede generar para las empresas. Pero no hay reputación sin imagen, ni imagen sin identidad corporativa por tanto hay que empezar a trabajar por la identidad a la hora de gestionar la reputación corporativa (Mut Camacho, 2010).

El concepto de público llega a su máxima amplitud con el término «stakeholders» se entiende como todos aquellos públicos que tienen intereses recíprocos con una empresa y que mantienen una relación estable con ella. Los más frecuentes son los empleados, los grandes clientes, los accionistas, las autoridades públicas y reguladores, los proveedores y «partners», los organismos de certificación, etc. y la imagen corporativa 
será configurada en la mente de estos a través de todos los «inputs» que les llegan, sean o no sean comunicativos. Por tanto, se requiere una actividad comunicativa organizada, lo que ha fomentado no sólo la aparición de la comunicación institucional sino la «complejización» de todo el proceso (Costa, 2008).

En conclusión, la comunicación corporativa deberá gestionar la totalidad de los recursos de comunicación y los activos intangibles de los que dispone una organización para llegar efectivamente a sus públicos (Garrido, 2001). En esta gestión jugará un papel fundamental la identidad corporativa en primera instancia y el objetivo último será que la imagen corporativa sea una síntesis fidedigna de la identidad corporativa (Costa, 2004). Para alcanzar este objetivo habrá que gestionar a su vez tanto el comportamiento, la cultura corporativa y la marca bajo el concepto marca experiencia. Finalmente una vez alcanzados los objetivos citados se pretende convertir la imagen positiva en reputación corporativa fin último de la comunicación corporativa.

\subsection{La gestión estratégica de la comunicación corporativa}

Esta investigación presenta al «Dircom» como el único y máximo exponente responsable de la gestión de la comunicación corporativa, aunque la realidad empresarial demuestra que es una figura profesional en proceso de implantación. Su entorno de trabajo debe ser el departamento de comunicación, que gestiona de forma conjunta e integrada los tres ámbitos de comunicación en las organizaciones: la comunicación corporativa, la comunicación interna y la comunicación de producto o marca. Sin embargo, hoy en día, la comunicación interna y la comunicación de producto o marca se encuentran en manos del departamento de recursos humanos y de marketing respectivamente, esto, junto a la falta de coordinación, dificulta un estilo propio de comunicación empresarial. La función esencial del Dircom es la consecución de una imagen positiva para la empresa, que mantenida en el tiempo de forma coherente, se convierta en buena reputación. Sus funciones más importantes por tanto, son el diseño de la estrategia de comunicación corporativa, y el control de la imagen, para lo que es fundamental que este profesional se encuentre junto al «management» de la compañía, en la cúpula directiva del organigrama (Costa, 2008).

De forma paralela a esta visión del Dircom, en esta investigación se describe un modelo de gestión de comunicación ideal al que cualquier empresa debe aspirar independientemente de su tamaño y estructura. Este modelo tomado como guía desarrolla la gestión estratégica de la imagen corporativa en tres etapas fundamentales, la primera, consiste en la definición de la estrategia de imagen donde como punto de partida se debe analizar la imagen que la empresa tiene en el momento de comenzar el trabajo, la segunda, la configuración de la personalidad corporativa que debe ser adaptada a la imagen intencional y finalmente, la propia gestión de la comunicación, que trata de controlar la imagen a través de distintos programas de comunicación. Para la propia implantación y desarrollo del modelo de gestión de la comunicación corporativa se plantean siete pasos fundamentales (Villafañe,1999:36):

1.- Auditoría de imagen.

2.- Observatorio permanente de imagen corporativa.

3.- Programas de identidad visual. 
4.- Manual de gestión de la comunicación.

5.- Programa de cambio cultural.

6.- Programas de comunicación corporativa.

7.- Programas de comunicación interna.

8.- Programa de comunicación de producto y marca.

Durante todo el desarrollo, que hace esta investigación sobre el modelo de gestión de la comunicación corporativa, se intenta realizar una aproximación continua a los cambios más recientes que están teniendo lugar, tanto en la sociedad como en el ámbito de la comunicación, para conocer las últimas tendencias que condicionan todo el discurso. Una de las más destacables es, cómo está cambiando el papel de los distintos públicos, principalmente condicionado por la aparición de la web 2.0, algo que está conduciendo hacia una nueva comunicación empresarial donde el escuchar y conversar, debe sustituir al tan practicado discurso empresarial que aspira a salir en prensa del modo más favorable (García Orosa, 2005).

\subsection{Particularidades de la gestión de la comunicación en pymes. Aproximación al anunciante valenciano y a los sectores tradicionales de la Comunidad Valenciana}

Los sectores objeto de estudio de esta investigación son mayoritariamente pymes y esta peculiaridad condiciona dicha gestión. Además, se profundiza en el anunciante valenciano, revisando si también posee particularidades dignas de reseña y del mismo modo, en los sectores tradicionales protagonistas de este estudio. Por tanto, la aproximación será desde lo más general, las pymes, hasta los clusters concretos sobre los que se está investigando y que como ya se ha concretado son: agroalimentación, calzado, cerámica, iluminación, juguete, mueble y textil.

\subsubsection{Investigaciones previas}

En relación a la caracterización específica de los sectores tradicionales de la Comunidad Valenciana se debe destacar que existen pocas investigaciones específicas sobre la gestión que hacen de su comunicación publicitaria y menos sobre la corporativa. Sí que hay algunos informes sectoriales o investigaciones académicas que hacen mención a algunos de los clusters pero siempre tratan el tema de manera indirecta. No obstante, aglutinando toda la información aparecida en diferentes fuentes, como el estudio de la Cámara de Valencia en 2004, se puede concluir que estos sectores pese a tener un número muy elevado de marcas y de anunciantes, generan un volumen de inversión publicitaria muy pequeño, además, se produce una elevada concentración de las acciones de comunicación en muy pocos soportes, principalmente en revistas especializadas, que las técnicas de comunicación utilizadas son publicitarias y que no hay programas integrales de comunicación. 


\subsubsection{Características básicas de la gestión de la comunicación en las pymes}

Las características básicas de la gestión de la comunicación que realizan las pymes (Carrillo, 2005) y que vienen condicionadas, por el ajuste que hacen a su volumen de negocio y a sus posibilidades, son las siguientes:

a.- Las pymes comunican y conocen las herramientas tradicionales de la comunicación comercial, especialmente publicidad, promociones, marketing directo $\mathrm{y}$ comunicación en el punto de venta, y aunque desconocen las últimas tendencias en la materia, sí están familiarizadas con la gestión de la comunicación publicitaria o comercial.

b.- Son muy exigentes con la rentabilidad de la comunicación.

c.- Al ser empresas de tamaño pequeño, perciben mayor riesgo a hacer alguna acción de comunicación que no dé resultados.

d.- El punto fuerte de sus acciones de comunicación es el contacto personal que pueden mantener con cada colectivo.

e.- El control personal de su negocio les lleva a querer ser autosuficientes y no contar con profesionales externos.

f- El escaso conocimiento de lo que significa comunicación empresarial, lleva a que en la mayor parte de los casos, lo relacionen exclusivamente con publicidad y ofertas comerciales.

g.- Aquellos que apuestan por llevar a cabo acciones publicitarias, apuestan por mensajes racionales apoyados, en la mayoría de casos, en el propio producto.

\subsubsection{Los públicos de las pymes}

Los públicos más relevantes y casi exclusivos para la pequeña y mediana empresa, son los externos, especialmente los clientes directos (Carrillo, 2005). Después están en un segundo grupo, por orden de preferencia las asociaciones empresariales donde deben estar presentes puesto que también lo están los competidores y el asociacionismo, les fortalece y acrecienta su representatividad. También destacan en el segundo grupo, los medios de comunicación, por su capacidad de generar opinión pública, aunque este tipo de empresas no se relacione con ellos directamente, sí los tienen en consideración a la hora de citarlos. Y finalmente, las administraciones públicas por su función reguladora y limitadora en muchos casos. En tercer puesto destacan, los accionistas de la empresa, los empleados y las entidades financieras. Y en cuarto y último lugar, citan a las asociaciones de consumidores e instituciones que cumplen un papel relevante a nivel social y económico. Este orden de priorización de los públicos de la empresa, poco tiene que ver con la gestión de intangibles como por ejemplo, el capital intelectual, donde los empleados son de los públicos más destacados. 


\subsubsection{La inversión publicitaria}

Respecto a la inversión publicitaria en medios convencionales del anunciante valenciano en 2008 (últimos datos disponibles en Infoadex, 2009) se concluye que ésta ha ido perdiendo peso en el ranking nacional de anunciantes (apenas llega al 3\% de la inversión nacional) en los dos últimos años, pérdida que viene potenciada por la bajada de inversión de los sectores tradicionales de la comunidad. En el ranking de los principales anunciantes valencianos, los sectores tradicionales de la Comunidad Valenciana que históricamente han sido los anunciantes más relevantes de la comunidad, apenas suponen en el año 2006, un $40 \%$ del total, dando mayor protagonismo a otros sectores como el de servicios, por ejemplo. Centrándose en los rasgos de la inversión publicitaria que realizan los siete sectores tradicionales de la comunidad, destaca principalmente el sector del juguete que concentra el $46 \%$ del total de la inversión, seguida de lejos por el sector de la agroalimentación, con un $15 \%$ de la inversión y después, el mueble, textil y cerámica con $12 \%, 11 \%$ y $10 \%$ respectivamente. Finalmente está el sector del calzado que supone el $6 \%$ de la inversión y el de la iluminación cuya inversión es casi inapreciable. Lo curioso es que el sector juguetero valenciano está compuesto por 147 empresas, frente a otros, como el textil, mueble, calzado en los que las inversiones son muy inferiores y cada uno está compuesto por unas casi 2000 empresas.

\section{Objetivos, hipótesis, metodología y trabajo de campo}

\subsection{Objetivos}

El objetivo general de esta investigación es conocer la consideración que los sectores tradicionales valencianos tienen acerca de la función de la comunicación corporativa en el seno de sus organizaciones.

De este gran objetivo surgen varios objetivos específicos.

$1^{\mathrm{o}}$.- Revelar la composición, estructura, expectativas y posición de los departamentos de comunicación de los sectores tradicionales valencianos.

$2^{\circ}$.- Reconocer cuales son las principales funciones del profesional de comunicación así como su formación.

\subsection{Hipótesis}

De acuerdo con los precedentes expuestos, esta investigación se plantea la siguiente hipótesis:

La hipótesis de esta investigación afirma que:

Los denominados sectores empresariales tradicionales mantienen una visión simple y reducida sobre las posibilidades de la función de la comunicación corporativa en el contexto actual. Depositan esta responsabilidad en las áreas de gerencia, marketing y comercial sin otorgar recursos o jerarquía a dicha función, ni implantando la figura del 
Dircom, y reduciendo su a la comunicación de producto. Esta perspectiva no permite generar una imagen positiva sostenida y, por tanto, una buena reputación para sus organizaciones.

\subsection{Metodología}

Para llevar a cabo esta investigación se ha elegido el modelo de investigación propuesto por el catedrático Rafael López Lita (2001) en la publicación Las Agencias de Publicidad: evolución y posicionamiento futuro. Así, el presente trabajo se articula en tres grandes etapas que se explica a continuación:

\subsubsection{Marco Teórico: fase documental.}

Se presenta todo el marco teórico y documental que sienta el punto de partida de la investigación. En él se revisa la comunicación corporativa como activo estratégico empresarial, la gestión estratégica de la comunicación corporativa, las particularidades de la gestión de la comunicación en pymes en los sectores tradicionales de la comunidad valenciana y la competitividad empresarial en estos sectores. Cuestiones que se han revisado de forma sintética en el inicio de este artículo.

\subsubsection{Marco Empírico: trabajo de campo.}

La siguiente etapa de la investigación consiste en el desarrollo del trabajo de campo. A continuación se describe la metodología empleada, el universo, la muestra seleccionada y las técnicas de investigación empleadas, todas ellas de corte cualitativo, a lo largo de las tres sub-etapas del trabajo de campo que concluyeron en 2010. Concretamente, en el trabajo de campo I se desarrollan 7 entrevistas, en el trabajo de campo 28 entrevistas y en el trabajo de campo III se celebran dos grupos focales. Todas ellas basadas en técnicas de investigación cualitativa (Álvarez-Gayou Jurgenson, 2003)

El trabajo de campo I y II pretende realizar una aproximación en profundidad al objeto de estudio de esta investigación, los sectores tradicionales de la Comunidad Valenciana y a cómo gestionan su comunicación corporativa, ofreciendo la visión de los protagonistas directos. Después de la reunión en marzo de 2006 con el Secretario General de la CIERVAL (Confederación de Organizaciones Empresariales de la Comunidad Valenciana), D. Rafael Montero para determinar cuáles eran los sectores objeto de esta investigación, se consideraron el sector de la agroalimentación, el sector del calzado, el sector de la cerámica, el sector de la iluminación, el sector del juguete, el sector del mueble y el sector textil, que incluye tanto textil-hogar prioritariamente, como textil-moda.

Trabajo de campo I y II

Para alcanzar el objetivo propuesto a trabajar con estos sectores, se establecieron dos grupos potenciales a los que investigar:

- En un primer lugar (equivale al trabajo de campo I), a los Secretarios Generales de las Asociaciones Empresariales representativas de los siete sectores tradicionales de 
la Comunidad Valenciana.

- En un segundo lugar (equivale al trabajo de campo II), a un número determinado de empresas de cada uno de los sectores objeto de estudio.

Una vez determinado el universo de estudio de esta investigación, tanto a nivel cualitativo como cuantitativo, se describe la selección de la muestra que los representa en este trabajo de campo I y II. A pesar de que se trata de una muestra cualitativa de conveniencia, ante el gran número de empresas que conforman el universo de estudio, si se recuerda eran 8.313 en el año 2007, se plantea la conveniencia de realizar dos tipos de aproximaciones, una a través de las asociaciones que hablan en nombre de todos sus asociados y otra, directamente con las empresas de los sectores seleccionados.

$1^{a}$ Muestra del trabajo de campo I. Asociaciones representantes de los siete sectores seleccionados.

En este cuadro que a continuación se presenta, se refleja de cada sector, la asociación y la persona a la que se ha entrevistado a finales del 2006, además se añaden como datos relevantes el ámbito de actuación de la misma, ya que en algunas ocasiones representan sólo a la Comunidad Valenciana y en otras, son asociaciones que aunque están en la comunidad son de ámbito nacional. Y como dato cuantitativo se aporta el número de asociados que tienen y por tanto, al número de empresas a las que representan

Fig. 1 Muestra del trabajo de campo I.

\begin{tabular}{|c|l|}
\hline SECTOR TRADICIONAL CV & \multicolumn{1}{|c|}{ ASOCIACIÓN EMPRESARIAL } \\
\hline 1.- AGROALIMENTACIÓN & $\begin{array}{l}\text { FEDACOVA (Federación empresarial de } \\
\text { agroalimentación de la Comunidad Valenciana) } \\
\text { D. José Granell Pascual (Secretario General) } \\
\text { AMBTO ACTUACIÓN: Comunidad Valenciana. } \\
\text { No ASOCIADOS: 32 Asociaciones que representan } \\
\text { 2100 empresas. }\end{array}$ \\
\hline 2.- CALZADO & $\begin{array}{l}\text { AICE (Asociación de Industriales de calzado de } \\
\text { Elche) la } \\
\text { FICE (Fed.de Industriales de calzado de la } \\
\text { Comunidad Valenciana) } \\
\text { D. Pedro Méndez Reyes (Secretario General de } \\
\text { ábas asociaciones) } \\
\text { ÁMBITO ACTUACIÓN: Representa el sur de } \\
\text { provincia Alicante con AICE y Nacional con FICE. } \\
\text { N ASOCIADOS: 230 empresas asociadas en } \\
\text { AICE. }\end{array}$ \\
\hline
\end{tabular}




\begin{tabular}{|c|c|}
\hline 3.- CERÁMICA & $\begin{array}{l}\text { ASCER (Asociación Española de Fabricantes de } \\
\text { Azulejos y Pavimentos Cerámicos) } \\
\text { Dña. Ana Martínez (Responsable Comunicación } \\
\text { y Prensa) } \\
\text { ÁMBITO ACTUACIÓN: Nacional. } \\
N^{\circ} \text { ASOCIADOS: } 200 \text { empresas asociadas (de las } \\
\text { que la mayoría están concentradas en la Comunidad } \\
\text { Valenciana). }\end{array}$ \\
\hline 4.- ILUMINACIÓN & $\begin{array}{l}\text { ARVET (Agrupación de Exportadores de } \\
\text { Transformados - Aparatos Iluminación) } \\
\text { FEDAI (Federación Española de Asoc. de } \\
\text { Industriales y Exportadores de Aparatos de } \\
\text { Iluminación) } \\
\text { D. Vicente Martínez (Director de ARVET) } \\
\text { Dña. Ma Ángeles Muñoz (Directora de FEDAI) } \\
\text { ÁMBITO ACTUACIÓN: ARVET Com. Valenciana } \\
\text { y FEDAI nacional. No ASOCIADOS: } 100\end{array}$ \\
\hline 5.- JUGUETE & 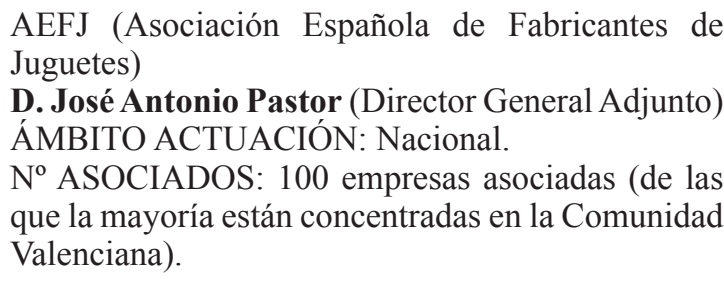 \\
\hline 6.- MUEBLE & $\begin{array}{l}\text { ANIEME (Asociación Nacional de Industriales y } \\
\text { Exportadores de Muebles de España) } \\
\text { Dña. Amparo Bertomeu (Directora General) } \\
\text { ÁMBITO ACTUACIÓN: Nacional } \\
\mathrm{N}^{0} \text { ASOCIADOS: } 250 \text { empresas asociadas (de las } \\
\text { que la mayoría están concentradas en la Comunidad } \\
\text { Valenciana). }\end{array}$ \\
\hline 7.- TEXTIL & $\begin{array}{l}\text { ATEVAL (Asociación de Empresarios Textiles de la } \\
\text { Comunidad Valenciana) } \\
\text { D. José Serna (Secretario General) } \\
\text { ÁMBITO ACTUACIÓN: Comunidad Valenciana. } \\
\text { No ASOCIADOS: } 530 \text { empresas asociadas. }\end{array}$ \\
\hline
\end{tabular}

$2^{a}$ Muestra, trabajo de campo II. Selección de cuatro empresas de cada uno de los siete sectores.

La segunda muestra para la realización del trabajo de campo II se obtiene en las 
entrevistas con los Secretarios Generales o Directores Generales de las asociaciones entrevistadas. A ellos se les solicita después de la entrevista, que nos den los datos y el contacto, siempre que lo consideren oportuno, de las empresas de su sector que consideren representan el término medio de su sector a nivel de desarrollo en comunicación corporativa. Cuando se les solicita este dato, la entrevista ya ha finalizado, y son conscientes de cuáles pueden ser las empresas que representen la media del sector en este tema. Se pidió a los entrevistados que citarán unas tres o cuatro empresas en esta situación intermedia y se decidió, que de cada sector se seleccionarían cuatro en total, que sumando los siete sectores daban como resultado veintiocho empresas a estudiar, cifra que se consideró suficientemente representativa. Además, en la investigación científica, y más específicamente en la cualitativa, según apunta Juan Luis ÁlvarezGayou (2003), llega un momento que se produce un efecto de saturación, en el que durante la obtención de información ésta empieza a ser igual, repetitiva o similar, así que por más amplia que sea la muestra, parece muy poco probable que la información cambie sustancialmente.

Fig. $2 N^{o}$ de empresas que representa la muestra seleccionada de asociaciones.

\begin{tabular}{|c|c|c|c|c|}
\hline $\begin{array}{l}\text { SECTOR } \\
\text { EMPRESARIAL }\end{array}$ & $\begin{array}{l}\text { ASOCIACIÓN Y } \\
\text { SU ÁMBITO DE } \\
\text { ACTUACIÓN }\end{array}$ & $\begin{array}{l}\text { TOTAL N }^{\circ} \\
\text { EMPRESAS } \\
\text { ASOCIADAS }\end{array}$ & $\begin{array}{l}\text { No EMPRESAS } \text { QUE } \\
\text { REPRESENTAN } \\
\text { NO COM. VAL. }\end{array}$ & $\begin{array}{l}N^{0} \text { EMPRESAS } \\
\text { QUE } \\
\text { REPRESENTAN } \\
\text { COM. VAL. }\end{array}$ \\
\hline Agroalimentación & $\begin{array}{l}\text { FEDACOVA } \\
\text { Com. Val. }\end{array}$ & 2100 & 0 & 2100 \\
\hline Calzado & $\begin{array}{l}\text { AICE } \\
\text { Sur Alicante } \\
\text { FICE } \\
\text { Nacional }\end{array}$ & $\begin{array}{l}230 \text { (no en el } \\
\text { total) } \\
\mathbf{2 5 8 4} \\
(66 \% \text { C.Val. }) \\
\end{array}$ & $\begin{array}{l}0 \\
879\end{array}$ & $\begin{array}{l}1705 \text { (incluidas } \\
\text { las } 230 \text { de AICE) }\end{array}$ \\
\hline Cerámica & $\begin{array}{l}\text { ASCER } \\
\text { Nacional } \\
\end{array}$ & $\begin{array}{l}200 \\
(95 \% \text { C.Val.) }\end{array}$ & 10 & 190 \\
\hline Iluminación & $\begin{array}{l}\text { FEDAI } \\
\text { Nacional }\end{array}$ & $\begin{array}{l}92 \\
(60 \% \text { C. Val })\end{array}$ & 37 & 55 \\
\hline Juguete & $\begin{array}{l}\text { AEFJ } \\
\text { Nacional }\end{array}$ & $\begin{array}{l}\mathbf{1 0 0} \\
(56 \% \text { C. Val })\end{array}$ & 44 & 56 \\
\hline Mueble & $\begin{array}{l}\text { ANIEME } \\
\text { Nacional }\end{array}$ & $\begin{array}{l}238 \\
(57 \% \text { C. Val })\end{array}$ & 103 & 135 \\
\hline Textil & $\begin{array}{l}\text { ATEVAL } \\
\text { Com. Val. }\end{array}$ & 530 & 0 & 530 \\
\hline \multicolumn{2}{|c|}{ TOTALES $n^{0}$ empresas: } & 5844 & 1073 & 4771 \\
\hline
\end{tabular}

Por tanto se considera una muestra de conveniencia las cuatro empresas por cada uno de los siete sectores estudiados, en la que tres deben representar a la media a nivel de desarrollo en comunicación corporativa, según los representantes de las asociaciones empresariales y una de ellas, debe ser una empresa que esté a la cabeza del sector en los temas de comunicación y creación de imagen de marca. De este modo, se conocería el 
grado más alto de desarrollo en la gestión de comunicación corporativa que alcanzan los distintos sectores (destacadas en el cuadro que a continuación se incluye, en color) y la situación media de la mayoría de empresas (en el cuadro que a continuación se incluye en blanco y negro).

Con todos estos condicionantes, la muestra definitiva del trabajo de campo II aparece reflejada en la tabla III.

Fig. 3 Muestra del trabajo de campo II.

\begin{tabular}{|c|c|}
\hline SECTOR EMPRESARIAL CV & EMPRESA ENTREVISTADA \\
\hline \multirow{4}{*}{ 1.- AGROALIMENTACIÓN } & $\begin{array}{l}\text { (1) NICOLAU JAMONES } \\
\text { D. Enrique Nicolau (Gerente y Director de Producción y Calidad, } \\
\text { Presidente de la Asociación de Industrias Cárnicas de Valencia) }\end{array}$ \\
\hline & $\begin{array}{l}\text { (2) DULCESOL } \\
\text { D. Rafael Juan (Consejero Delegado) }\end{array}$ \\
\hline & $\begin{array}{l}\text { (3) INTERCITRUS(Organización InterprofesionalAgroalimentaria } \\
\text { del sector de la Naranja y el grupo Mandarinas) } \\
\text { Dña. Anabel Siguan (Directora Técnica) }\end{array}$ \\
\hline & $\begin{array}{l}\text { (4) CHOCOLATES VALOR } \\
\text { D. Pedro López (Director Gerente) }\end{array}$ \\
\hline \multirow{4}{*}{ 2.- CALZADO } & $\begin{array}{l}\text { (1) CALZADOS POLO SUR } \\
\text { Dña. Marian García (Comité Dirección y Dir. Comercial) }\end{array}$ \\
\hline & $\begin{array}{l}\text { (2) PANTER } \\
\text { D. Ramón Pajares (Gerente) }\end{array}$ \\
\hline & $\begin{array}{l}\text { (3) CALZADOS PAKER } \\
\text { D. Salvador Martínez (Gerente) } \\
\text { D. Francisco García (Gerente) }\end{array}$ \\
\hline & $\begin{array}{l}\text { (4) PIKOLINOS } \\
\text { D. Javier Soler (Adjunto Dir. Comercial y Comunicación) }\end{array}$ \\
\hline \multirow{4}{*}{ 3.- CERÁMICA } & $\begin{array}{l}\text { (1) CERÁMICA DECORATIVA } \\
\text { D. José Castellano (Director General) }\end{array}$ \\
\hline & $\begin{array}{l}\text { (2) KEROS } \\
\text { D. Joaquín Emilio Martí (Gerente) }\end{array}$ \\
\hline & $\begin{array}{l}\text { (3) GAYA } \\
\text { D. Luigi M. Limido (Director de Marketing) }\end{array}$ \\
\hline & $\begin{array}{l}\text { (4) TAU CERÁMICA, } \\
\text { D. Manuel Llopis (Director de Comunicación) }\end{array}$ \\
\hline
\end{tabular}




\begin{tabular}{|c|c|}
\hline \multirow{4}{*}{ 4.- ILUMINACIÓN } & $\begin{array}{l}\text { (1) LÁMPARAS RAFAEL TORMO } \\
\text { Dña. Amparo Tormo (Gerente) }\end{array}$ \\
\hline & $\begin{array}{l}\text { (2) SAVOY HOUSE } \\
\text { D. Francisco Albiñana (Director General) }\end{array}$ \\
\hline & $\begin{array}{l}\text { (3) ANTARES } \\
\text { D. Guillermo Simó (Director de Marketing) }\end{array}$ \\
\hline & $\begin{array}{l}\text { (4) ALMERICH } \\
\text { D. Antonio Almerich (Director Gerente) }\end{array}$ \\
\hline \multirow{4}{*}{ 5.- JUGUETE } & $\begin{array}{l}\text { (1) MINILAND } \\
\text { D. José Verdú (Director General) }\end{array}$ \\
\hline & $\begin{array}{l}\text { (2) MUNECAS ARIAS } \\
\text { D. Francisco Arias (Gerente) }\end{array}$ \\
\hline & $\begin{array}{l}\text { (3) JUGUETES FALOMIR } \\
\text { D. José María Castellano (Director Comercial) } \\
\text { Presidente de la Feria del Juguete de Valencia. }\end{array}$ \\
\hline & $\begin{array}{l}\text { (4) FÁBRICA DE JUGUETES CHICOS } \\
\text { D. Emilio Valls (Director Comercial) }\end{array}$ \\
\hline \multirow{4}{*}{ 6.- MUEBLE } & $\begin{array}{l}\text { (1) COLECCIÓN ALEXANDRA } \\
\text { D. Juan Ventura (Gerente) }\end{array}$ \\
\hline & $\begin{array}{l}\text { (2) CASANOVA GANDÍA } \\
\text { D. Pedro Casanova (Gerente) }\end{array}$ \\
\hline & $\begin{array}{l}\text { (3) PALANCA } \\
\text { D. José Carlos Palanca (Gerente) }\end{array}$ \\
\hline & $\begin{array}{l}\text { (4) ANDREU WORLD } \\
\text { D. José Palau (Director de Marketing y Ventas) }\end{array}$ \\
\hline \multirow{4}{*}{ 7.- TEXTIL } & $\begin{array}{l}\text { (1) REVERT } \\
\text { D. José Ramón Revert (Gerente) }\end{array}$ \\
\hline & $\begin{array}{l}\text { (2) BELPLA } \\
\text { D. José Belda (Gerente) }\end{array}$ \\
\hline & $\begin{array}{l}\text { (3) DOLORES CORTÉS } \\
\text { Dña. Dolores Font Cortés (Gerente) }\end{array}$ \\
\hline & $\begin{array}{l}\text { (4) AZNAR TEXTIL (BONDRAP) } \\
\text { Dña. Ana Aznar (Comité de Dirección y Dir. Marketing y } \\
\text { Comunicación) }\end{array}$ \\
\hline
\end{tabular}

Trabajo de campo III

Uno de los objetivos metodológicos de esta investigación era el llegar a retratar el objeto de estudio, a través de la utilización de diferentes métodos de investigación y con las perspectivas de todos los públicos implicados en la materia. Ya en el marco teórico se obtuvo la visión de los académicos que han investigado directa o indirectamente 
sobre estas cuestiones, en el trabajo de campo I y II se ha ofrecido la visión de los protagonistas directos, la de los sectores tradicionales de la Comunidad Valenciana. Pues bien, finalmente el siguiente paso metodológico pretende ser una aproximación al mismo objeto desde la visión de los agentes externos que colaboran en la gestión de la imagen y la comunicación corporativa de estos sectores, los profesionales de la comunicación valenciana, persiguiendo como principal objetivo la validación y contraste de los resultados del trabajo de campo I y II. tradicionales.

Han sido realizados por tanto, dos grupos focales en junio y julio de 2009, con representantes de las dos Juntas de Gobierno de los dos máximos órganos representantes de la profesión de la comunicación valenciana.

- Ilustre Colegio Oficial de Publicitarios y Relaciones Públicas de la Comunidad Valenciana.

- Asociación de Agencias de Publicidad de la Comunidad Valenciana (AAPCV).

El listado de participantes y cargos que tienen se enumera a continuación:

El primer grupo focal fue realizado el 29 de junio de 2009, en la propia sede de la AAPCV y tuvo una duración de 1 hora y ' 16 minutos. Los componentes de la junta de gobierno que asistieron fueron los que se especifican a continuación.

Componentes de la junta de gobierno de la aapcv que participaron en el grupo focal:

1.- D. Eugenio Martín, Presidente del Grupo Cuestión y Vicepresidente de la AAPCV.

2.- Dña. Carlota Serratosa, Consejera Delegada de Dimarco.

3.- D. Vicente Vañó, Director de Servicios al Cliente de Engloba.

4.- Y D. Eduard Farràn, Director Creativo Ejecutivo de Dimarco, que aunque no es miembro de la junta de gobierno de la AAPCV, sí tiene una vinculación estrecha con la asociación y vino en representación de su agencia.

El segundo fue realizado el 13 de julio de 2009, en la propia sede de Colegio Oficial de Publicitarios y Relaciones Públicas de la Com. Val. y la duración fue de 1 horas y ' 10 minutos. Los componentes de la junta de gobierno que asistieron fueron los que a continuación se detallan.

Componentes de la junta de gobierno del Colegio Oficial de Publicitarios y Relaciones Públicas de la Com. Val. que participaron en el grupo focal:

1.- Dña. Ana Portaceli, Socia de NGT y Presidenta del Colegio de Publicitarios.

2.- D. Antonio Xandri, Secretario General del Colegio Oficial de Publicitarios.

3.- Dña. Carlota de Dios, Directora General de Carlota de Dios y Asociados.

4.- D. José Segura, Director de Planificación Estratégica de Pértiga Branding.

5.- Dña. Isabel de Salas, Defensora del universitario y profesora de la Universidad CEU Cardenal Herrera.

6.- Dña. Marta Giner, Jefa Comercial y de Relaciones Institucionales de Aumar.

7.- D. Miguel Lucas, Director General de Método y Asociados. 


\section{Resultados de la investigación}

Los resultados del trabajo que cumplen los objetivos de la investigación de campo giran en torno a cinco ejes: la valoración y consideración que tienen a cerca de la comunicación así como sus expectativas y el análisis de la figura del Dircom, atendiendo a su ubicación en el organigrama, su formación o sus funciones.

\subsection{La consideración de la comunicación en los sectores tradicionales valencianos}

\subsubsection{En qué lugar sitúan la comunicación.}

Las empresas entrevistadas sitúan la responsabilidad de la comunicación en las empresas entorno a estas áreas:

$1^{\mathrm{a}}$.- En la mayoría de casos está en manos de la gerencia, concretamente en once de las empresas entrevistadas, con ciertos apoyos internos o externos para el trabajo diario.

$2^{\text {a }}$.- La segunda opción más frecuente es el departamento comercial. En ocho de las empresas entrevistadas.

$3^{\text {a }}$.- El departamento de marketing como tal está creado como tal en cuatro casos y en uno es una intención no materializada. Este departamento depende en la mitad de los casos de la dirección general y en la otra mitad de la propia dirección comercial.

De forma puntual también depende del departamento de producción y diseño y en un caso existe un departamento de publicidad y comunicación, ligado a comercial, y sin autoridad en la estrategia. Otra empresa, directamente no contempla la opción de un departamento de comunicación.

Por otra parte, en el polo de las asociaciones todos los entrevistados han necesitado diferenciar el tamaño de la empresa para situar esta actividad, ya que del tamaño depende en gran medida donde esté ubicada la comunicación empresarial en cada una de las organizaciones y no se han visto capaces de generalizar. En lo que sí han coincidido las siete asociaciones entrevistadas y que es relevante para las conclusiones de esta investigación, es que todas las compañías sitúan y ligan la actividad de comunicación directamente a la venta de producto, a la actividad comercial y al departamento o actividad de marketing, como sucede en el caso de las empresas entrevistadas, independientemente de que lo tengan o no implantado.

De este modo, al margen de esta primera clasificación se puede hacer una vinculación entre la dimensión de la empresa y la responsabilidad de la comunicación:

\section{$1^{\mathrm{a}}$.- Pequeñas y medianas empresas}

En este ámbito existe una gran confusión en cuanto a terminología y formación en comunicación. Así como en manos de la dirección general de la compañía, están prácticamente todas las funciones vitales (pedidos, compras, mantenimiento, producción, etc.) entre ellas está, lógicamente, las decisiones notables a nivel de comunicación. Hay quienes afirman, dos de las siete entrevistas realizadas a asociaciones, que esta situación se dará siempre y cuando se contemple esta actividad en la empresa, porque la realidad es que en el $99 \%$ de los casos, ni siquiera se tiene en consideración por parte de la dirección la función de la comunicación. 
$2^{\mathrm{a}}$.- Grandes empresas

También en este nivel la comunicación es considerada como actividad dependiente de marketing pero dentro de esta tipología de empresas ya hay algunas (dos, tres o cuatro en función del sector del que se hable) que tienen departamento propiamente dicho. Lo que no hay en ningún caso es un departamento específico de comunicación. En el caso de tener responsable de marketing, dan por supuesto que es porque la dirección general está involucrada y concienciada de su necesidad y por tanto, la actividad de comunicación está al mismo nivel en importancia que la actividad comercial, la financiera, la de producción, etc.

\subsection{2. ¿Gasto o inversión? Qué beneficios que puede reportar una buena gestión de la comunicación.}

La inmensa mayoría de los entrevistados afirma que es una inversión, veintiuna empresas concretamente de las veintiocho. Pero destacan lo difícil que es conocer el retorno de la inversión y medir qué resultados tiene, como sucede en otras áreas donde es más sencillo. Respecto a los que afirman que es un gasto, cinco de las veintiocho entrevistas realizadas dicen que te sube los costes de producción y que el retorno que obtienen es nulo. También cabe destacar a tres entrevistados que no lo tienen claro y dudan si es gasto o inversión, de hecho uno afirma que la mitad considera que es un gasto y la otra mitad una inversión.

Respecto a la pregunta de qué les reporta, se debe destacar que ésta, es una de las preguntas que más ha costado a los entrevistados tanto empresas como a asociaciones contestar, de hecho pese a afirmar que son muchos los beneficios que reporta la correcta gestión de la comunicación, no acababan de verbalizarlos o detallarlos claramente.

La respuesta más habitual ha sido la necesidad de «darte a conocer o tener presencia en el mercado», incluso se ha afirmado que simplemente debes estar por necesidad ya que si no tienes una presencia a nivel de comunicación no existes y que por ello, es necesario el apoyar el recuerdo del producto en cada acción que lleva a cabo la empresa.

En segundo lugar se ha citado el beneficio de «vender» o de alcanzar mayor volumen de ventas. Además, argumentan que esto es fácil de medir con las estadísticas de venta que tienen las empresas, con lo que pueden verificar con mayor facilidad lo que les reporta.

A la par, aparecen beneficios como «alcanzar la imagen deseada por la empresa y obtener poder frente a la distribución». El primero queda argumentado como una necesidad de comunicar lo que eres como compañía y lo que realmente estás haciendo si quieres como empresa que tenga una imagen tuya.

Otro de los argumentos mencionados es la ventaja de «diferenciarte» $\mathrm{o}$ «posicionarte» a través de la correcta gestión de la comunicación, pero sólo ha sido mencionado por dos de los entrevistados.

\subsection{3. ¿Tienen en cuenta que todo comunica?}

Otra cuestión que revela la consideración sobre la comunicación es la consciencia de la permanente emisión de mensajes en una organización, ya sea de forma intencionada 
o no. De las veintiocho entrevistas realizadas dieciséis afirman que sí que es algo por lo que se preocupan, puesto que es fundamental porque una mala gestión en este sentido puede estropear el esfuerzo realizado durante muchos años. No obstante, quitando a cuatro de estos dieciséis, que afirman con seguridad tener muy controladas estas cuestiones, el resto, la inmensa mayoría, confiesan la dificultad que entraña para sus empresas el poder transmitir y controlar esto. La mayoría afirma que es muy complicado acertar ya que supone un cambio de cultura complicado, sobre todo cuando se trata de empresas que han sufrido varios cambios generacionales de dirección. Unos tres entrevistados en cambio afirman les ha ayudado mucho la implantación de la ISO de Calidad para la que han tenido que realizar encuestas de satisfacción del personal y otro tipo de protocolos que han ayudado a implantar esta filosofía.

Por el contrario, nueve de los entrevistados afirman no tener en cuenta esto, principalmente porque el personal de la empresa no vive la organización como la dirección, no están involucrados. Incluso hay quien afirma que a los empleados sólo les interesa ir a fichar, trabajar lo justo e irse y eso sí, cobrar a final de mes.

Y en dos casos ha sido imposible obtener una respuesta coherente ante esta pregunta, por mucho que se ha intentado reconducir la entrevista.

\subsection{La figura del Dircom}

\subsection{1 ¿Tienen responsable de comunicación? En caso negativo ¿Por qué no lo tienen?}

Responsable de comunicación como tal, tan sólo tiene una de las empresas entrevistadas, el cargo exacto es Responsable de Comunicación y Publicidad. Hay dos empresas que introducen el término comunicación en el cargo pero que la relacionan con otros temas como en un caso con Diseño y Comunicación y en el otro Marketing y Comunicación. Tres empresas afirman que no hay una sola persona que asuma estas tareas sino que quedan diluidas o repartidas entre varios puestos.

Tan sólo una de las compañías entrevistadas había afirmado que nadie asumía estas tareas, y ya habían dado como motivo principal el que no es un área prioritaria para ellos. Sin embargo, en tres de los entrevistados que afirmaron que el tema lo llevaba gerencia directamente, se denotó en la propia conversación que eran temas que no contemplaban en principio, y al formularles esta pregunta, contestaron los tres que es un gasto muy elevado para una pequeña empresa con falta de recursos y que se encargan ellos del tema igual que gestionan muchas más áreas. También uno de ellos afirmó que no hay una cultura de comunicación en su organización ya que tienen otros intereses.

\subsection{2. ¿Dónde está ubicado en el organigrama?}

Las respuestas sobre esta ubicación giran en torno a varios espacios, de forma paralela al punto 4.1.1:

$1^{a}$.- En la mayoría de casos está en manos de la gerencia, con apoyos internos o externos para el trabajo diario. 
$2^{\mathrm{a}}$.- Segunda opción más frecuente departamento comercial o coordinador de ventas. $3^{\text {a }}$.- Departamento de marketing.

$4^{a}$.- Departamento de producción y diseño.

5 a.- Sólo un caso tiene departamento de comunicación y publicidad.

\subsection{3 ¿Qué formación tiene?}

La formación más habitual de la persona que asume las tareas de comunicación es la comercial, seguida de dirección empresarial o económicas, lo que respalda que los departamentos desde los que se gestionan estos temas son gerencia y comercial principalmente. En cuatro casos se apunta que la formación es en marketing y estrategias, y en otros tres casos, en bellas artes, artes y oficios y diseño. De manera puntual tan sólo citado en un caso hay quien tiene la formación de arquitectura, en otro caso de químico y otro en idiomas.

Destaca una empresa que afirma que se preocupan por la formación interna en estos temas y apunta que para ello se dan clases al personal encargado de la comunicación de oratoria, «escaparatismo», promoción de producto, etc.

4.2.4. ¿Cuáles son las cuatro funciones principales que realiza este profesional? ¿Qué porcentaje de sus labores son estratégicas y qué otro porcentaje son labores ejecutivas? (a empresas).

Como es habitual las contestaciones a esta pregunta se muestran por orden de aparición, en función del número de veces que han sido citadas de mayor a menos importancia. Por mayoría es la «realización de catálogos y folletos» y la «asistencia a ferias», las tareas principales del profesional que asume el área de comunicación, en la opinión de los entrevistados. En tercer lugar aparece lo que es el «contacto directo y la comunicación con el cliente» a la que se da la misma importancia que a la elaboración del «dossier de prensa»y «relaciones con los medios» para conseguir apoyo redaccional. Después esta la «comunicación en el punto de venta» y la «publicidad» aparece al mismo nivel, destacando en publicidad el llevar el contacto directo con la revista.

Citado por cuatro de los entrevistados destacan como una de las labores la definición de la «estrategia de comunicación» y en un caso hablan de «plan de marketing». Y ya de manera aislada, citado por uno o dos de los entrevistados aparecen las siguientes funciones: Posicionamiento de marca, creación de muestrarios para la venta, creación de nuevos productos, boletines de comunicación interna, «packaging», patrocinios deportivos, gestión de la página web, patrocinios, formación específica para clientes y gestión de quejas.

Las funciones habituales de este profesional mixto son aquellas que tienen que ver con la comunicación comercial: «realización de catálogos y folletos», la «asistencia a ferias», el «contacto directo y la comunicación con el cliente». El «dossier de prensa» o las «relaciones con los medios», funciones clásicas de la comunicación corporativa, quedan en un segundo plano. 


\section{Conclusiones}

No hay lugar a dudas que el contexto actual de la sociedad de la información exige organizaciones responsables, transparentes que sean capaces de conciliar los intereses privados y los intereses de la comunidad, de los «stakeholders». Vivimos en el paradigma del siglo XXI que según Costa (2003) viene determinado por los valores intangibles (cultura, identidad, comunicación e imagen) como superación del modelo de producción que ha imperado hasta finales del siglo XX. El nuevo modelo se superpone al anterior y la antiguas coordenadas (producción, finanzas, marketing y organización) se jerarquizan frente a las nuevas. Trasladado al mercado significa que el la imagen y la reputación las variables que permiten reconocer y discriminar unos productos y servicios sobre otros en una sobreoferta.

Así, la asunción de la comunicación corporativa como un activo estratégico empresarial es una realidad en las grandes empresas cotizadas de este país y del mundo. Dentro de todo este cambio de filosofía organizacional se asume que toda actuación de la empresa está comunicando, incluida la inactividad en este terreno, por tanto, la comunicación corporativa se convierte en un activo estratégico empresarial que debe ser gestionado y que en caso de una disposición bidireccional de la información con los distintos públicos, se obtendrá una imagen positiva sostenida en el tiempo o sea una buena reputación.

Una buena gestión de la comunicación corporativa obliga a asumir ciertas premisas. Por ejemplo, la existencia de un director de comunicación que coordine los distintos mensajes y aporte coherencia entre los discursos y los públicos, que maneje los tres ámbitos de comunicación en las organizaciones: la comunicación corporativa, la comunicación interna y la comunicación de producto o marca. Es imprescindible su ubicación en la cúspide la organización y la adopción de un perfil eminentemente estratégico, máxime con la aparición de la web 2.0, algo que está conduciendo hacia una nueva comunicación empresarial donde la base se da en la conversación.

No obstante, los sectores tradicionales valencianos no han llegado todavía a esa consideración estratégica de la función de la comunicación. Tal y como se exponía en el marco teórico, los «clusters» valencianos son Pymes con unas debilidades evidentes en materia de comunicación. La hipótesis expuesta de forma previa a la investigación que proponía que estos sectores plantean una visión simple y reducida sobre la comunicación corporativa, visión que se ha visto confirmada tras el trabajo de campo. Por tanto, se puede decir que los sectores de agroalimentación, calzado, cerámica, iluminación, juguete, mueble y textil en territorio valenciano siguen un modelo vetusto, arraigado en el siglo XX y que no asumen la función de la comunicación como un activo estratégico relegando al Dircom a un segundo plano o más bien a la inexistencia.

Esta conclusión se alcanza tras el planteamiento de líneas de estudio: la composición, estructura, expectativas y ubicación de los departamentos de comunicación de los sectores tradicionales valencianos; y el reconocimiento de las figura del Dircom en estos sectores.

En la primera línea queda evidenciado que la atención hacía la comunicación en los sectores tradicionales valencianos no es prioritaria. La investigación revela que no existen departamentos específicos de comunicación, que se asigna esta responsabilidad principalmente al área de gerencia o en segundo lugar al área comercial o de marketing. 
Si se hace una distinción entre pymes y grandes empresas se encuentra que en las Pymes ni siquiera se tiene en consideración por parte de la dirección la función de la comunicación mientras que en las grandes empresas existe tal función pero queda vinculada a otro departamento o gerencias. Nunca de forma independiente.

Es relevante que todas las compañías sitúan y ligan la actividad de comunicación directamente a la venta de producto, a la actividad comercial y al departamento o actividad de marketing, como sucede en el caso de las empresas entrevistadas, independientemente de que lo tengan o no implantado. De hecho, la respuesta más habitual ante los beneficios de una buena gestión de la comunicación ha sido la necesidad de «darte a conocer o tener presencia en el mercado» y en segundo lugar se ha citado el beneficio de "«vender» o de alcanzar mayor volumen de ventas.

Sin embargo, cuestiones vinculadas a la consecución de activos intangibles como «alcanzar la imagen deseada por la empresa» o «diferenciarte» y «posicionarte» son consideradas de menor relevancia.

La segunda línea de estudio relacionada con la propia figura del Dircom en estos sectores. Este perfil profesional no existe en puridad y en el mejor de los casos se comparte con otras funciones (marketing, diseño o comercial). Depende en la mayoría de los casos de gerencia, del departamento comercial o de marketing. Sólo un caso tiene departamento de comunicación y publicidad y en ningún caso ocupa una posición jerárquica o estratégica.

La formación más habitual de la persona que asume las tareas de comunicación es la comercial, seguida de dirección empresarial o económicas, lo que respalda que los departamentos desde los que se gestionan estos temas son gerencia y comercial principalmente. En cuatro casos se apunta que la formación es en marketing y estrategias, y en otros tres casos, en bellas artes, artes y oficios y diseño. De manera puntual tan sólo citado en un caso hay quien tiene la formación de arquitectura, en otro caso de químico y otro en idiomas.

Finalmente, se puede concluir los sectores objeto de estudio no conocen y, por tanto, no confían en las posibilidades de una función estratégica de la comunicación en el seno de sus organizaciones. Rechazan la idea de que la imagen corporativa, correctamente gestionada, es un activo estratégico al servicio de cualquier organización, prevaleciendo la visión cortoplacista y la función comercial y de marketing. Cuestiones que tendrán que revisar si desean ser competitivos en el futuro inmediato.

\section{Bibliografía}

Alvarez-Gayou Jurgenson, J. L (2003): Cómo hacer una investigación cualitativa, fundamentos y metodología, México, Paidós Ecuador.

Bel Mallén, J. I. (coord.) (2004): Comunicar para crear valor. Dirección de comunicación en las organizaciones, Pamplona, EUNSA.

Benlloch Osuna, M,T. Y López Lita, R. (2005): «La marca territorio. El marketing de ciudad, una herramienta al servicio de las marcas territorio», 99\%, 3, 45-49, Castellón, Ed. Grupo Mibalia.

- (2006): «De la marca comercial a la marca territorio», Revista Recerca, 18, 116-139,Castellón, Revista de Pensament i Anàlisi. 
Blay Arráez, R. Y Benlloch Osuna, M,T (2006) «¿Qué puede hacer la comunicación por el sector? » Periódico El mundo Castelló al día, Castellón, 22 de febrero de 2006, 8, Suplemento Cevisama.

- (2007): «Análisis de la comunicación en los sectores relevantes para la provincia de Castellón». 99\%. Castellón, 5, 23-27, Castellón, Ed. Grupo Mibalia.

- (2010): «La gestión de la imagen y la comunicación corporativa en las pequeñas y medianas empresas valencianas» en Lopéz Lita, R y Mut Camacho, M. (Coord) Gestión de intangibles. Importancia en el desarrollo territorial, Castellón, Ed. Ciencias Sociales, 66-89.

Cámara Oficial De Comercio, Industria Y Navegación De Valencia (2004): «La nueva economía global y su incidencia en los sectores tradicionales de la Comunidad Valenciana», Valencia, Informe elaborado por la Cámara de Comercio.

CAPriotti, P. (1992): La imagen de la empresa. Estrategia para una comunicación integrada. Barcelona, Consejo Superior de Relaciones Públicas de España.

CArrillo, M.V. et al. (2005): Imagen y comunicación en las pymes, Madrid, Editorial Fragua.

Costa, J. (2001): La comunicación en acción. Informe sobre la nueva cultura de la gestión, Barcelona, Paidós.

- (2003): Imagen corporativa en el siglo XXI, La Crujía, Buenos Aires.

- (2004): La imagen de marca. Un fenómeno social, Barcelona, Paidós.

- (2008): El dircom hoy, Barcelona, Costa Punto Com Editor.

García Orosa, B. (2005): Los altavoces de la actualidad: radiografía de los gabinetes de comunicación. Madrid, Netbiblo.

Garrido, F. J. (2001): Comunicación estratégica. Las claves de la comunicación empresarial en el siglo XXI. Barcelona, Gestión 2000.

Generalitat Valenciana (2009): «II Planes sectoriales de competitividad», nota de prensa emitida por el IMPIVA el 5 de enero de 2009, Valencia. Fecha consulta: 5 septiembre de 2009. www.impiva.es,

Giuliani, E. Y Rabellotti, R. (Edit.) (2005): Clusters facing competition: the importance of external linkages, Londres, Ashgate.

INFOADEX (2009). Resumen estudio Infoadex de la inversión publicitaria 2009. Madrid. Fecha consulta: 1 junio de 2009.

LóPez LitA, R. (2000): La comunicación: la clave del bienestar social, Madrid, Ediciones El Drac.

- (2001): Las agencias de publicidad: evolución, posicionamiento y futuro, Castellón, Publicaciones de la Universitat Jaume I.

Mut Camacho, M. (2010): «Revisión de intangibles territoriales que contribuyen al avance socioeconómico», Pensar la publicidad, Vol. IV, nº 1, 117-128.

Pérez GonzÁlez, R.A. (2006): Estrategias de comunicación, Barcelona, Ariel.

Pizzolante Negrón, I. (2006): El poder de la comunicación estratégica. Apuntes de un evangelizador corporativo, Caracas, El Nacional.

Pla Barber, J. Y Otros (2007): «Crisis, actitudes directivas y estrategia en los sectores manufactureros tradicionales: el sector textil español», Universia Business Review, Segundo Trimestre 2007, 68-83. 
R. Blay - M. Benlloch - G. Sanahuja La percepción sobre la función de la comunicación corporativa

Santesmases Mestre, M. (1996): Términos de marketing, diccionario y base de datos, Madrid, Pirámide.

Villafañe, J. (1999): La gestión profesional de la imagen corporativa, Madrid, Pirámide.

- (2004): La buena reputación. Claves del valor intangible de las empresas, Madrid, Pirámide. 